\title{
Effect of systemic antihypertensives on change in intraocular pressure after initiating topical prostaglandins for primary open-angle glaucoma
}

This article was published in the following Dove Medical Press journal:

Clinical Ophthalmology

\author{
Mehdi Siddiquil,* \\ Joshua Iltis ${ }^{1, *}$ \\ Petar Yanev' \\ John Sladic' \\ Charles Huynh' \\ Daniel Nolan ${ }^{2}$ \\ Michael Singer ${ }^{2}$ \\ 'Long School of Medicine, University \\ of Texas Health San Antonio, San \\ Antonio, TX, USA; ${ }^{2}$ Medical Center \\ Ophthalmology Associates, San \\ Antonio, TX, USA \\ *These authors contributed equally \\ to this work
}

\begin{abstract}
Purpose: There is a limited understanding of factors that influence the efficacy of topical glaucoma medication. Our study is a long-term, case-control analysis of how systemic antihypertensive (anti-HTN) medications influence the change in IOP after initiating prostaglandin (PG) drop therapy.
\end{abstract}

Materials and methods: A retrospective chart review of 3,781 patients was performed on patients with a diagnosis of glaucoma suspect that progressed to primary open-angle glaucoma (POAG) by ICD-9 codes over a 10-year period. Inclusion criteria consisted of the following: 1) progression from preglaucoma to glaucoma diagnosis in a time span of $\geq 6$ months; 2) two visual fields recorded between these dates; 3 ) initial average IOP of both eyes of $\geq 21 \mathrm{mmHg}$; and 4) initiation of topical PG therapy alone. IOP (in $\mathrm{mmHg}$ ) was measured at initiation of PG drops and at next visit.

Results: One hundred eleven patients were qualified for analysis. Patients not on anti-HTN agents had an average IOP decrease of $6.38 \pm 0.56 \mathrm{mmHg}$. Comparatively, patients on anti-HTN agents had an average IOP decrease of $6.66 \pm 0.48 \mathrm{mmHg}(P=0.61)$. In addition, there was no statistical difference between IOP decrease between patients on single vs multiple systemic anti-HTN agents $(P=0.85)$. There were eight nonresponders to PGs on no anti-HTN medications and 12 nonresponders on anti-HTN medication $(P=0.55)$.

Conclusion: Systemic anti-HTN medication use did not significantly impact IOP reduction after topical PG initiation for POAG. Additionally, nonresponse to PG therapy was not correlated to systemic anti-HTN use.

Keywords: glaucoma, topical prostaglandins, IOP, systemic antihypertensives, ocular hypertension, prostaglandin eye drops

\section{Introduction}

Glaucoma, one of the leading causes of blindness in the world, is a disease in which damage to the optic nerve occurs due to increased IOP secondary to either increased production of aqueous humor or decreased outflow of aqueous humor. Glaucoma has many subclassifications including closed-angle glaucoma, neovascular glaucoma, and primary openangle glaucoma (POAG). POAG is the most common type of glaucoma in the USA., ${ }^{1,2}$

POAG is generally treated with topical eye drops such as alpha-2 agonists, betablockers, carbonic anhydrase inhibitors, and prostaglandins (PGs). Beta-blockers, alpha-2 agonists, and carbonic anhydrase inhibitors work by decreasing aqueous humor secretion into the anterior chamber of the eye. PGs increase aqueous drainage by increasing outflow through the scleral canal. Patients with glaucoma commonly have other chronic comorbid conditions, notably hypertension, diabetes, and migraines,
Correspondence: Michael Singer Medical Center Ophthalmology Associates, 9157 Huebner Road, San Antonio, TX 78240, USA

Tel +I 2102693754

Email msingerII@me.com 
which also happen to be risk factors. PGs are first-line agents in treating POAG since they have shown the highest efficacy in decreasing IOP. ${ }^{2-4}$

Recent studies have examined the effects of systemic antihypertensive beta-blocker therapy administered concurrently with topical beta-blocker glaucoma therapy and have identified a smaller decrease in IOP compared with topical beta-blocker therapy alone. Concurrent treatment with systemic antihypertensive beta-blocker therapy has also been shown to augment the efficacy of brimonidine, an alpha 2 agonist, in lowering IOP.,5,6 These findings suggest that certain systemic antihypertensive agents may have either a synergistic or antagonistic effects with certain kinds of topical glaucoma therapy.

There is currently a limited understanding of which factors influence the efficacy of topical PG medication in patients with POAG. This study was designed to identify the relationship between the use of different systemic antihypertensive medications and the subsequent change in IOP when initiating topical PG therapy in patients with newly diagnosed POAG. Although current literature has described some ways in which systemic antihypertensive medications affect the progression of glaucoma, there is very limited information about how these medications influence the efficacy, or magnitude of decrease in IOP, of topical PG therapy. ${ }^{6-9} \mathrm{~A}$ better understanding of these interactions would be a valuable supplement to clinical decision making, efficiency, and time management, as well as disease treatment and overall outcomes. ${ }^{10-15}$

\section{Materials and methods}

A retrospective chart review of 3,781 patients was performed on patients with a diagnosis of glaucoma suspect that progressed to open-angle glaucoma by ICD-9 codes over a 10 -year period. This retrospective review was approved by Specialty Surgery Center IRB prior to the start of the study. The patient data were gathered using the electronic medical record at Medical Center Ophthalmology Associates in San Antonio, Texas, USA. All patients signed a consent form that allowed their protected health information to be used. All stages of this study were conducted in accordance with the principles set forth by the Declaration of Helsinki. Criteria for inclusion in this study were as follows: 1) a patient must have an initial IOP of $\geq 21 \mathrm{mmHg}$ measured with Tono-Pen AVIA tonometer before initiation of topical PG medication; 2) treatment for POAG should consists of topical PG therapy alone; 3 ) patients must have demonstrated progression from glaucoma suspect to diagnosis of POAG according ICD-9 codes on chart review over a span of 6 months or longer; 4) the patient must have at least two visual field tests on record between the time of glaucoma suspect diagnosis and POAG diagnosis. The rationale for this last criterion was to further support correct diagnosis of POAG. Diagnosis of POAG was made clinically with the help of a combination of optical coherence tomography (OCT), tonometry, visual field, and fundus examination. The topical PG prescribed to treat POAG in the patient population studied at our institution is latanoprost $0.005 \%(50 \mathrm{mg} / \mathrm{mL})$ daily. These inclusion criteria were implemented to give more legitimacy to our results by ensuring patients were properly worked up in the same clinic with visual field testing, were being followed chronically and consistently for progression of glaucoma suspect to POAG, and by keeping an IOP cutoff consistent with commonly accepted IOP cutoff for POAG diagnosis. ${ }^{16,17}$

Patient charts that met inclusion criteria were evaluated for demographic data, diagnosis of hypertension, systemic antihypertensive medications, and IOP measurements $(\mathrm{mmHg})$ at the initiation date of topical PG therapy for POAG. IOP at the subsequent visit following initiation of therapy was then recorded. The difference in IOPs at the dates of treatment initiation and at the subsequent visits was used to calculate average change in IOP. Antihypertensive medications were reviewed at the time of initiating PG drop and subsequent visit to control consistency in blood pressure control and were included if there was no change in medications. Data were evaluated to compare average change in IOP between patients on no systemic antihypertensive medications, single systemic antihypertensive therapy, and multiple systemic antihypertensives. A paired $t$-test was used to evaluate for significant change in IOP between these three groups.

\section{Results}

One hundred and eleven patients met the inclusion criteria for analysis. The average IOP drop after initiating PG therapy was $6.60 \mathrm{mmHg}$. Our demographic breakdown was composed of $43.2 \%$ males and $56.8 \%$ females. Of that group, $58.6 \%$ were Caucasian, $34.2 \%$ were Hispanic, and 7.2\% were of another ethnicity. In this population, $59.5 \%$ of patients had a previous diagnosis of hypertension, while $40.5 \%$ did not. The age range of our patient population was 38-94 years ( median $=70$, mean $=68.7)$ (Table 1$)$.

Once PG therapy was initiated, patients not on antihypertensive agents $(\mathrm{N}=40)$ had an average IOP decrease of $6.38 \pm$ $0.56 \mathrm{mmHg}$, while patients on single antihypertensive agents $(\mathrm{N}=40)$ had an average IOP decrease of $6.66 \pm 0.48 \mathrm{mmHg}$. 
Table I Patient demographic data broken down by gender, ethnicity, hypertension diagnosis, and age

\begin{tabular}{|c|c|}
\hline \multicolumn{2}{|c|}{ Patient demographics $(\mathrm{N}=\mathrm{I} I \mathrm{I})$} \\
\hline Characteristics & $\mathrm{N}(\%)$ \\
\hline \multicolumn{2}{|l|}{ Gender } \\
\hline Male & $48(43)$ \\
\hline Female & $63(57)$ \\
\hline \multicolumn{2}{|l|}{ Ethnicity } \\
\hline Caucasian & $65(59)$ \\
\hline Hispanic & $38(34)$ \\
\hline Others & 8 (7) \\
\hline \multicolumn{2}{|c|}{ Hypertension diagnosis } \\
\hline Yes & $71(64)$ \\
\hline No & $40(36)$ \\
\hline \multicolumn{2}{|l|}{ Age (years) } \\
\hline Median & 70 \\
\hline Range & $38-94$ \\
\hline
\end{tabular}

Patients on multiple antihypertensive agents $(\mathrm{N}=31)$ had an average IOP decrease of 6.82 $.68 \mathrm{mmHg}$ (Table 2).

There was no significant difference between the magnitude of IOP decrease between patients on no systemic antihypertensive medications vs a single antihypertensive medication $(P=0.61)$. In addition, there was no statistical difference between IOP decrease when comparing patients on single vs multiple systemic antihypertensive agents $(P=0.85$; Table 3$)$.

In addition, we compared IOP decrease in terms of class of antihypertensives. No significant difference was found in IOP decrease in all these groups.

The number of nonresponders (IOP reduction $<10 \%$ ) to PG drops was split with eight patients in the group not on antihypertensive medications and 12 patients in the group who were taking systemic antihypertensives. A chi-squared analysis between these two groups showed no significant difference between proportion of nonresponders in patients on antihypertensives and patient who were not $(P=0.55)$.

\section{Discussion}

The relationship between hypertension and glaucoma remains unclear. Several studies have found that systemic hypertension, vasospasm, and acute hypotension are potential risk factors for glaucoma, ${ }^{18-22}$ while other studies have found no association between hypertension and $\mathrm{OAG}^{23,24} \mathrm{In}$ a study by Klein et al, blood pressure and IOP levels were shown to be correlated and IOP levels are a risk factor for developing glaucoma in most populations. ${ }^{25}$ When it comes to treatment, IOP is the only modifiable factor in POAG. ${ }^{26}$ Logically, it seems that by lowering a patient's blood pressure, the IOP should decrease and the rate of glaucomatous damage reduce as well; however, the relationship between blood pressure and glaucoma progression is confounded by its effect on IOP. This along with other mechanisms may explain why many conflicting studies exist on the influence that blood pressure-lowering agents have on glaucoma progression. For instance, taking antihypertensives at night may worsen glaucoma because both the antihypertensives and a person's circadian rhythm contribute to lowering of blood pressure to a level where ischemia in ocular blood vessels can occur. ${ }^{27}$ Taking these factors into consideration, it is unclear how much of a role antihypertensives play in glaucoma progression.

PGs are used to treat glaucoma because they effectively lower IOP with simple dosing and limited side effects. ${ }^{28}$ By lowering the IOP of the eye, PGs help to slow or inhibit further damage to the optic nerve caused by high pressures in the

Table 2 Comparison of mean decrease in IOP following prostaglandin drop therapy isolating different classes of antihypertensive medications

\begin{tabular}{|l|l|l|l|l|}
\hline $\begin{array}{l}\text { Antihypertensive } \\
\text { class }\end{array}$ & $\begin{array}{l}\text { Number of patients } \\
\text { taking drug }\end{array}$ & $\begin{array}{l}\text { Baseline IOP } \\
(\mathbf{m e a n} \pm \mathbf{S E}, \mathbf{m m H g})\end{array}$ & $\begin{array}{l}\text { IOP decrease from baseline } \\
\text { IOP }(\mathbf{m e a n} \pm \mathbf{S E}, \mathbf{m m H g})\end{array}$ & $\begin{array}{l}\text { Mean percentage reduction } \\
\text { from baseline IOP }(\mathbf{m e a n} \pm \mathbf{S E})\end{array}$ \\
\hline No drugs & 40 & $23.724 \pm 0.445$ & $6.566 \pm 0.572$ & $27.2 \pm 2.0$ \\
\hline ACE inhibitors & 30 & $23.767 \pm 0.413$ & $6.250 \pm 0.568$ & $25.8 \pm 2.1$ \\
\hline ARB & 26 & $23.712 \pm 0.478$ & $6.712 \pm 0.687$ & $28.0 \pm 2.7$ \\
\hline BB & 17 & $23.088 \pm 0.410$ & $6.500 \pm 0.881$ & $27.6 \pm 3.4$ \\
\hline Thiazides & 15 & $24.333 \pm 0.654$ & $7.467 \pm 0.919$ & $30.2 \pm 3.4$ \\
\hline CCB & 16 & $23.813 \pm 0.559$ & $7.188 \pm 1.113$ & $29.5 \pm 4.5$ \\
\hline Loop diuretic & 4 & $22.750 \pm 1.109$ & $9.250 \pm 2.454$ & $39.8 \pm 9.0$ \\
\hline
\end{tabular}

Notes: The majority of these patients were on multiple antihypertensive medications of different classes. No statistical difference was found in mean IOP decrease between different antihypertensive drug classes.

Abbreviations: ACE, angiotensin-converting enzyme; ARB, angiotensin II receptor blocker; BB, beta-blocker; CCB, calcium channel blocker; SE, standard error. 
Table 3 No statistically significant difference was found in IOP decrease following starting topical prostaglandins with regard to the number of different antihypertensive drug classes the patients were taking at the initiation of prostaglandin drop therapy

\begin{tabular}{|c|c|c|c|c|}
\hline \multicolumn{5}{|c|}{ Change in IOP after initiating prostaglandin therapy } \\
\hline Medication regimen & $\begin{array}{l}\text { Average pretreatment IOP } \\
\text { (mmHg, both eyes) }\end{array}$ & $\begin{array}{l}\text { Average posttreatment IOP } \\
\text { ( } \mathrm{mmHg} \text {, both eyes) }\end{array}$ & $\begin{array}{l}\text { Average decrease } \\
\text { in IOP }\end{array}$ & P-value \\
\hline $\begin{array}{l}\text { No systemic antihypertensive } \\
\text { medications }\end{array}$ & 23.72 & 17.39 & 6.34 & \multirow[t]{2}{*}{0.61} \\
\hline $\begin{array}{l}\text { Any systemic antihypertensive } \\
\text { medications }\end{array}$ & 23.72 & 17.02 & 6.70 & \\
\hline $\begin{array}{l}\text { Single systemic antihypertensive } \\
\text { medications }\end{array}$ & 23.79 & 17.19 & 6.60 & \multirow[t]{2}{*}{0.85} \\
\hline $\begin{array}{l}\geq 2 \text { systemic antihypertensive } \\
\text { medications }\end{array}$ & 23.63 & 16.81 & 8.82 & \\
\hline
\end{tabular}

eye. With contradictory reports on the efficacy of IOP reduction in systemic antihypertensives, and with the existence of people who are poor responders to PG therapy, ${ }^{29}$ this study was able to look at any differences that may exist between patients on PGs solely and those also on antihypertensives. Other studies have looked at outcomes in patients taking both systemic medications and PGAs. A Canadian study found that patients who were taking antihypertensives while on PGAs were less likely to need additional drop therapy compared with patients just taking PGAs. This suggests a possible synergistic effect in glaucoma treatment between topical PGs and systemic medications. ${ }^{30}$ The report did not look at IOP reduction directly like in our study, but instead looked for the need for adjunctive drop therapy as a proxy for glaucoma progression; however, the reasons for why an adjunctive IOP reducing drop was added were not detailed in the study. Because our study looked directly at magnitude of IOP reduction when systemic antihypertensives are taken along with PG, our results show that antihypertensives are unlikely to influence the efficacy of PGAs. By eliminating variables such as concurrent medication use, we can better understand which factors truly affect the efficacy of topical PG drops.

A recent study published in the American Academy of Ophthalmology (AAO) found that certain systemic medications influenced the progression of POAG. The AAO paper studied the relationship between systemic medications and the likelihood of a required procedure for POAG. It was found that three separate medication classes had statistically significant results. ${ }^{9}$ Systemic beta-blockers were found to have a protective effect on POAG; thus, patients on these medications required fewer procedures. It has long been known that systemic beta-blockers can lower IOP, and thus, these medications may provide some benefit to disease progression. ${ }^{3,6}$ It has also been shown that systemic medications like beta-blockers can impact the efficacy of topical drop medications such as timolol which are used to lower IOP. ${ }^{3}$ The study also found that Selective serotonin reuptake inhibitors (SSRIs) may have a protective benefit in POAG progression. Patients on SSRIs were $30 \%$ less likely to require a procedure pertaining to POAG than nonusers of SSRI. ${ }^{9}$ On the contrary, calcium channel blockers (CCBs) were shown to actually increase progression to POAG and these patients required more ocular procedures than patients not on CCBs. According to this study, patients on CCB were $26 \%$ more likely to require a procedure due to their POAG than patients not on CCBs. ${ }^{9}$

Studies such as these are very intriguing and open up the possibilities of systemic medication affecting progression of POAG. While these studies are finding interesting correlations, there have been no studies showing adequate explanations for these findings. Furthermore, there are certain weaknesses to these studies mentioned because it is not known what variables each ophthalmologist is looking at when deciding surgical management. There is lack of uniformity between each ophthalmologist's decision making that can lead to skewed results. This is where our project can add value to these unexplained findings. If ophthalmologists are deciding to move forward with procedures based on IOP readings, our research shows that systemic antihypertensive use will not falsely alter IOP readings in patients on PG drops. Currently, there is very little knowledge of factors influencing PG therapy. Our study shows that systemic antihypertensive medications do not alter the IOP reduction between patients taking antihypertensives and patients not taking any antihypertensives. Our study also shows that there is no alteration in IOP reduction between patients taking one antihypertensive versus taking multiple antihypertensives. This study allows us to eliminate certain variables from our decision making when we consider those that are influencing POAG progression. By showing that systemic antihypertensives have no 
effect on IOP reduction in patients on PG therapy, it suggests that another pathologic process is at work in patients described in the AAO study. For instance, patients on CCB may have a higher risk of POAG progression, but this risk would not be attributed to the lack of efficacy of PG drops in reducing IOP.

An important circumstance we explored was patients not responding to PG therapy. It is not understood what contributes to a patient's nonresponse. It has been shown that $\sim 15 \%$ of patients put on PG therapy are nonresponders. ${ }^{28}$ There currently is no widely acceptable definition of nonresponders to PG drops; however, we looked at multiple studies that used $<10 \%$ reduction in IOP as a cutoff for their nonresponders..$^{29,31}$ Going by this definition, we found that the number of nonresponders (IOP reduction $<10 \%$ ) to PG drops was split with eight patients $(21 \%)$ in the group not on antihypertensive medications and 12 patients (15\%) in the group that were taking systemic antihypertensives. Our data are consistent with studies we looked at which showed approximately a $14 \%-15 \%$ nonresponse rate in their patient population. ${ }^{29}$ The fact that there was no statistically significant difference between the total number of nonresponders in the group of patients on antihypertensives versus the group of patients not taking antihypertensives means that nonresponse to PG drop therapy supports the notion that this phenomenon is independent of a medication interaction involving antihypertensives. This is important because a substantial number of patients $(\sim 15 \%)$ do not respond to PG therapy, which is the first-line treatment for lowering IOP.

Previous research has found that ophthalmologists spend $27 \%$ of each patient visit time entering information into the electronic health records (EHRs), equating to an average of 3.7 hours per day. ${ }^{1}$ Research has shown that less time spent on EHR could result in improved clinical efficiency and improved patient satisfaction. ${ }^{10-15}$ The implication of these results is that ophthalmologists may not need to worry about the effects of different antihypertensive medications on patients when prescribing PG therapy for the treatment of POAG. Alleviating concern about a patient's antihypertensive medications, ophthalmologists can prescribe PGs knowing that its efficacy or nonresponse is not attributed to the patient's concurrent antihypertensive use. In addition, primary care physicians who are prescribing antihypertensive medications do not have to worry about decreasing PG IOP-lowering effects.

For now, more research needs to be done in order to better understand the disease process of glaucoma. There are too many contradictions in the literature for us to be sure of what associations exist between systemic medications and glaucoma. Our study supports the notion that systemic antihypertensives did not affect the efficacy of topical PG drops and are not factors in patients who are nonresponders to PG.

\section{Limitations}

This study has limitations associated with monitoring diagnosis, treatment, and disease progression of POAG. For one, although the inclusion criteria attempted to ensure that all patients would be on a similar stage of POAG progression, there was variability in IOP at the time of diagnosis, which may allow for a wide range of posttreatment IOP. Additionally, the main indicator that was followed in this study to gauge the efficacy of glaucoma treatment was change in IOP. In reality, this is not as important of a marker of glaucoma disease state as OCT imaging or visual field testing. In addition, follow-up appointments for evaluation of efficacy of PG therapy occurred at variable time after diagnosis. Regarding assessing the effects of both systemic antihypertensive therapy and PG therapy, it is possible that noncompliance with either antihypertensives or PGs may have skewed results.

A study in AAO published in July 2018 showed negative effects of CCBs and positive effects of SSRIs on progression of POAG. ${ }^{9}$ Our results did not show decreased effect of CCB on PG IOP-lowering effect. In addition, this was a single-center study that may have led to regional biases. Also, we did not account for patients on SSRIs or serotoninnorepinephrine reuptake inhibitors, which have been shown to alter progression of POAG in certain recently published studies.

Medical Center Ophthalmology Associates (MCOA) does not regularly record blood pressure values for their patients, so we were not able to correlate IOP with blood pressure. This is a limitation because some studies have shown that blood pressure can influence IOP. ${ }^{25}$

Finally, we did not distinguish between the different PG eye drops available. There is a debate on the difference in efficacy of different PG drops. One study showed that bimatoprost had more clinically significant IOP-lowering effect than tafluprost, latanoprost, and travaprost. ${ }^{29,32}$ Another study showed no statistical difference in efficacy between drops. ${ }^{33}$

\section{Conclusion}

Systemic antihypertensive medication use did not affect the magnitude of IOP reduction in patients with newly diagnosed POAG who were treated with topical PG therapy. Additionally, nonresponse to PG therapy was not correlated to systemic antihypertensive use. 


\section{Data sharing statement}

Data were gathered from MCOA Electronic Medical Records and due to patient confidentiality, are not publicly available.

\section{Acknowledgment}

This paper was presented at The Association for Research in Vision and Ophthalmology (ARVO) April 2018 and not published elsewhere.

\section{Author contributions}

All authors contributed to data analysis, drafting and revising the article, gave final approval of the version to be published, and agree to be accountable for all aspects of the work.

\section{Disclosure}

Michael Singer is a consultant for Allergan, Allegro, Ampio, Genentech, Ophthotech, Regeneron, Santen, Aerpio, Optos, Alcon, Clearside, Notal Vision, Guidepoint, and Alimera. The other authors report no conflicts of interest in this work.

\section{References}

1. Boland MV, Ervin AM, Friedman DS, et al. Comparative effectiveness of treatments for open-angle glaucoma: a systematic review for the U.S. Preventive Services Task Force. Ann Intern Med. 2013;158(4):271-279.

2. Maier PC, Funk J, Schwarzer G, Antes G, Falck-Ytter YT. Treatment of ocular hypertension and open angle glaucoma: meta-analysis of randomised controlled trials. BMJ. 2005;331(7509):134.

3. Schuman JS. Effects of systemic beta-blocker therapy on the efficacy and safety of topical brimonidine and timolol. Brimonidine Study Groups 1 and 2. Ophthalmology. 2000;107(6):1171-1177.

4. Li F, Huang W, Zhang X. Efficacy and safety of different regimens for primary open-angle glaucoma or ocular hypertension: a systematic review and network meta-analysis. Acta Ophthalmol. 2018;96(3):e277-e284.

5. Epstein DL, Krug JH, Hertzmark E, Remis LL, Edelstein DJ. A longterm clinical trial of timolol therapy versus no treatment in the management of glaucoma suspects. Ophthalmology. 1989;96(10):1460-1467.

6. Khawaja AP, Chan MP, Broadway DC, et al. Systemic medication and intraocular pressure in a British population: the EPIC-Norfolk eye study. Ophthalmology. 2014;121(8):1501-1507.

7. Gottfredsdottir MS, Allingham RR, Shields MB. Physicians' guide to interactions between glaucoma and systemic medications. J Glaucoma. 1997;6(6):377-383.

8. Zheng W, Dryja TP, Wei Z, et al. Systemic medication associations with presumed advanced or uncontrolled primary open-angle glaucoma. Ophthalmology. 2018;125(7):984-993.

9. Müskens RP, de Voogd S, Wolfs RC, et al. Systemic antihypertensive medication and incident open-angle glaucoma. Ophthalmology. 2007; 114(12):2221-2226

10. Read-Brown S, Hribar MR, Reznick LG, et al. Time requirements for electronic health record use in an academic ophthalmology center. JAMA Ophthalmol. 2017;135(11):1250-1257.

11. Lam JG, Lee BS, Chen PP. The effect of electronic health records adoption on patient visit volume at an academic Ophthalmology Department. BMC Health Serv Res. 2016;16(7):7.

12. Poissant L, Pereira J, Tamblyn R, Kawasumi Y. The impact of electronic health records on time efficiency of physicians and nurses: a systematic review. J Am Med Inform Assoc. 2005;12(5):505-516.

13. Pandit RR, Boland MV. The impact of an electronic health record transition on a glaucoma subspecialty practice. Ophthalmology. 2013;120(4): 753-760.
14. Chan P, Thyparampil PJ, Chiang MF. Accuracy and speed of electronic health record versus paper-based ophthalmic documentation strategies. Am J Ophthalmol. 2013;156(1):165-172.

15. Alkureishi MA, Lee WW, Lyons M, et al. Impact of electronic medical record use on the Patient-doctor relationship and communication: a systematic review. J Gen Intern Med. 2016;31(5):548-560.

16. Bonomi L, Marchini G, Marraffa M, Morbio R. The relationship between intraocular pressure and glaucoma in a defined population. Ophthalmologica. 2001;215(1):34-38.

17. Nitta K, Wajima R, Tachibana G, et al. Prediction of visual field progression in patients with primary open-angle glaucoma, mainly including normal tension glaucoma. Sci Rep. 2017;7(1):15048.

18. Bonomi L, Marchini G, Marraffa M, Bernardi P, Morbio R, Varotto A. Vascular risk factors for primary open angle glaucoma: the EgnaNeumarkt study. Ophthalmology. 2000;107(7):1287-1293.

19. Wilson MR, Hertzmark E, Walker AM, Childs-Shaw K, Epstein DL. A case-control study of risk factors in open angle glaucoma. Arch Ophthalmol. 1987;105(8):1066-1071.

20. Tielsch JM, Katz J, Sommer A, Quigley HA, Javitt JC. Hypertension, perfusion pressure, and primary open-angle glaucoma. A populationbased assessment. Arch Ophthalmol. 1995;113(2):216-221.

21. Topouzis F, Coleman AL, Harris A, et al. Association of blood pressure status with the optic disk structure in non-glaucoma subjects: the Thessaloniki eye study. Am J Ophthalmol. 2006;142(1):60-67.

22. Leske MC, Wu SY, Hennis A, Honkanen R, Nemesure B, BESs Study Group. Risk factors for incident open-angle glaucoma: the Barbados eye studies. Ophthalmology. 2008;115(1):85-93.

23. Hulsman CA, Vingerling JR, Hofman A, Witteman JC, de Jong PT. Blood pressure, arterial stiffness, and open-angle glaucoma: the Rotterdam study. Arch Ophthalmol. 2007;125(6):805-812.

24. Deb AK, Kaliaperumal S, Rao VA, Sengupta S. Relationship between systemic hypertension, perfusion pressure and glaucoma: a comparative study in an adult Indian population. Indian J Ophthalmol. 2014;62(9): 917-922.

25. Klein BE, Klein R, Knudtson MD. Intraocular pressure and systemic blood pressure: longitudinal perspective: the Beaver dam eye study. $\mathrm{Br}$ J Ophthalmol. 2005;89(3):284-287.

26. Ocular Hypertension Treatment Study Group, European Glaucoma Prevention Study Group, Gordon MO, et al. Validated prediction model for the development of primary open-angle glaucoma in individuals with ocular hypertension. Ophthalmology. 2007;114(1):10-19.

27. Krasińska B, Karolczak-Kulesza M, Krasiński Z, et al. Effects of the time of antihypertensive drugs administration on the stage of primary open-angle glaucoma in patients with arterial hypertension. Blood Press. 2012;21(4):240-248.

28. Toris CB, Gabelt B'ann T, Kaufman PL. Update on the mechanism of action of topical prostaglandins for intraocular pressure reduction. Surv Ophthalmol. 2008;53(6):S107-S120.

29. Inoue K, Setogawa A, Tomita G. Nonresponders to prostaglandin analogs among normal-tension glaucoma patients. J Ocul Pharmacol Ther. 2016;32(2):90-96.

30. Iskedjian M, Walker JH, Desjardins O, et al. Effect of selected antihypertensives, antidiabetics, statins and diuretics on adjunctive medical treatment of glaucoma: a population based study. Curr Med Res Opin. 2009;25(8):1879-1888.

31. Kawaguchi I, Higashide T, Ohkubo S, Kawaguchi C, Sugiyama K. Comparison of efficacy of four prostaglandin analogues by bilateral treatment in healthy subjects. Jpn J Ophthalmol. 2012;56(4):346-353.

32. Ranno S, Sacchi M, Brancato C, Gilardi D, Lembo A, Nucci P. A prospective study evaluating IOP changes after switching from a therapy with prostaglandin eye drops containing preservatives to nonpreserved tafluprost in glaucoma patients. ScientificWorldJournal. 2012;2012: 804730 .

33. El Hajj Moussa WG, Farhat RG, Nehme JC, et al. Comparison of efficacy and ocular surface disease index score between bimatoprost, latanoprost, travoprost, and tafluprost in glaucoma patients. J Ophthalmol. 2018;2018:1-7. 


\section{Publish your work in this journal}

Clinical Ophthalmology is an international, peer-reviewed journal covering all subspecialties within ophthalmology. Key topics include: Optometry; Visual science; Pharmacology and drug therapy in eye diseases; Basic Sciences; Primary and Secondary eye care; Patien Safety and Quality of Care Improvements. This journal is indexed on

Submit your manuscript here: http://www.dovepress.com/clinical-ophthalmology-journal

\section{Dovepress}

PubMed Central and CAS, and is the official journal of The Society of Clinical Ophthalmology (SCO). The manuscript management system is completely online and includes a very quick and fair peer-review system, which is all easy to use. Visit http://www.dovepress.com/ testimonials.php to read real quotes from published authors. 